\title{
CLIC status and outlook
}

\author{
Steinar Stapnes ${ }^{* \dagger}$ \\ CERN, Geneva \\ E-mail: steinar.stapnesecern.ch \\ Daniel Schulte \\ CERN, Geneva \\ E-mail: daniel.schultedcern.ch
}

The Compact Linear Collider study (CLIC) is in the process of completing a Conceptual Design Report for a multi-TeV linear electron-positron collider. The CLIC-concept is based on high gradient normal-conducting accelerating structures. The RF power for the acceleration of the colliding beams is produced by a novel two beam acceleration scheme, where power is extracted from a high current drive beam that runs parallel with the main linac. In order to establish the feasibility of this concept a number of key issues have been addressed. A short summary of the progress and status of the corresponding studies will be given.

The 2011 Europhysics Conference on High Energy Physics-HEP 2011,

July 21-27, 2011

Grenoble, Rhône-Alpes France

\footnotetext{
* Speaker.

On behalf of the CLIC collaboration.
} 


\section{Overview}

The fundamental CLIC parameters and the conceptual layout for the machine can be found in table 1 and in Fig. 1 below. More details about the CLIC machine are given in [1] and the CDR currently in preparation. This summary is a slightly shortened and adapted version of the proceedings submitted to IPAC 2011 [2].

The main (colliding) beams are produced in conventional electron and positron sources and accelerated to about $2.8 \mathrm{GeV}$. The beam emittances are reduced in a pre-damping ring followed by a damping ring. In the ring-to-main-linac transport system (RTML) the beams are compressed longitudinally and accelerated to $9 \mathrm{GeV}$. The main linac uses $100 \mathrm{MV} / \mathrm{m}, 12 \mathrm{GHz}$, normal conducting accelerating structures to achieve the final beam energy. In the beam delivery system (BDS) the beam is cleaned by collimation and compressed to a tiny size at the collision point.

The necessary RF power in for the main linac accelerating structures is extracted from a highcurrent, low-energy drive beam that runs parallel to the colliding beams and is generated in a central complex.

The most important design challenges of CLIC will be discussed in the following:

- The main linac gradient; an issues of the accelerating structures.

- The two beam concept, which is essential to provide the main linac RF power; i.e. the drive beam generation, PETS (power extraction and transfer structures), two beam module and the drive beam deceleration.

- The ultra low beam emittances and sizes to reach high luminosity. In particular alignment and stabilization of the main linac and BDS components.

- The machine protection system.

It should be noted that the CLIC parameters are the result of a full cost optimization [3], which took into account the main constraints on the RF structures and the beam dynamics.

\section{Main Linac Gradient}

Each main linac contains about $7000023 \mathrm{~cm}$-long accelerating structures and the total ratio of active length to linac length is almost $80 \%$. The structure design has been carefully optimised using empirical constraints to achieve a gradient of $100 \mathrm{MV} / \mathrm{m}$ [4]. The main limitation arises from so-called breakdowns, i.e. sparks that can occur in the structure during the RF pulse, which can give transverse kicks to the beam. The kick size will be measured in CTF3. Typically the breakdown probability $p$ increases with the gradient $G$ and pulse length $\tau$ as $p \propto G^{30} \tau^{5}$ [6]. We conservatively assume that a single breakdown in a main linac structure renders the beam pulse useless for luminosity. This should happen only in $1 \%$ of the beam pulses at the target gradient of $100 \mathrm{MV} / \mathrm{m}$, which results in a target breakdown rate of $\leq 3 \times 10^{-7} \mathrm{~m}^{-1} \mathrm{pulse}^{-1}$.

Four designs are tested: T18, TD18, T24 and TD24. TD24 corresponds to the CLIC structure; T24 is simplified by the absence of the damping wave guides. T18 and TD18 correspond to an earlier, less developed design, which would be less efficient; again " $D$ " indicates the presence of 


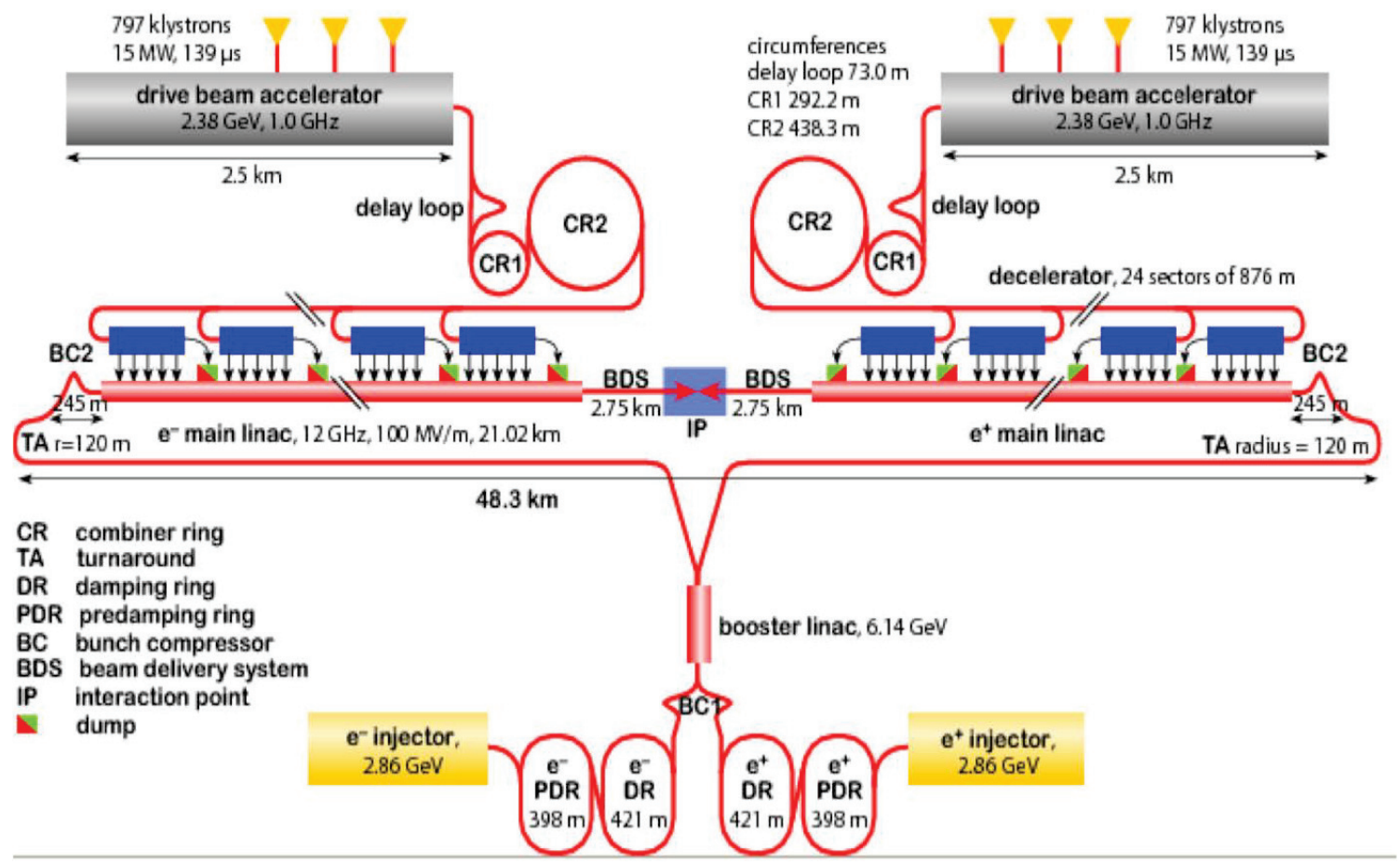

Figure 1: Conceptual layout of CLIC.

Table 1: Fundamental CLIC parameters. The luminosity quoted is within $1 \%$ of the nominal centre-of-mass energy.

\begin{tabular}{|c|c|c|}
\hline Centre-of-mass energy & 3 & $\mathrm{TeV}$ \\
Luminosity & $2 \times 10^{34}$ & $\mathrm{~cm}^{-2} \mathrm{~s}^{-1}$ \\
particles per bunch & $3.72 \times 10^{9}$ & \\
horizontal IP beam size & $\approx 40$ & $\mathrm{~nm}$ \\
vetical IP beam size & $\approx 1$ & $\mathrm{~nm}$ \\
bunches per pulse & 312 & \\
bunch separation & 0.5 & $\mathrm{~ns}$ \\
pulse rate & 50 & $\mathrm{~s}^{-1}$ \\
\hline
\end{tabular}

damping waveguides. At SLAC and KEK [7] klystrons with $11.424 \mathrm{GHz}$ are used and the structures have simply been scaled in all dimensions to the klystron frequency. At CERN $12 \mathrm{GHz}$ power can be produced in the CTF3 two-beam test stand (TBTS), and in the near future with a klystron currently being installed. However, in TBTS the low repetion rate does not allow condition the structure fully.

The tests have been performed with pulse lengths and breakdown rates similar to the CLIC parameters. The measurement data is shown in Fig. 2 together with the expected CLIC gradients, obtained by scaling the pulse length and breakdown rate to the the CLIC values using scaling formula above. Two T18 (built at SLAC and KEK) achieved an unloaded gradient of about 105 MV/m; 


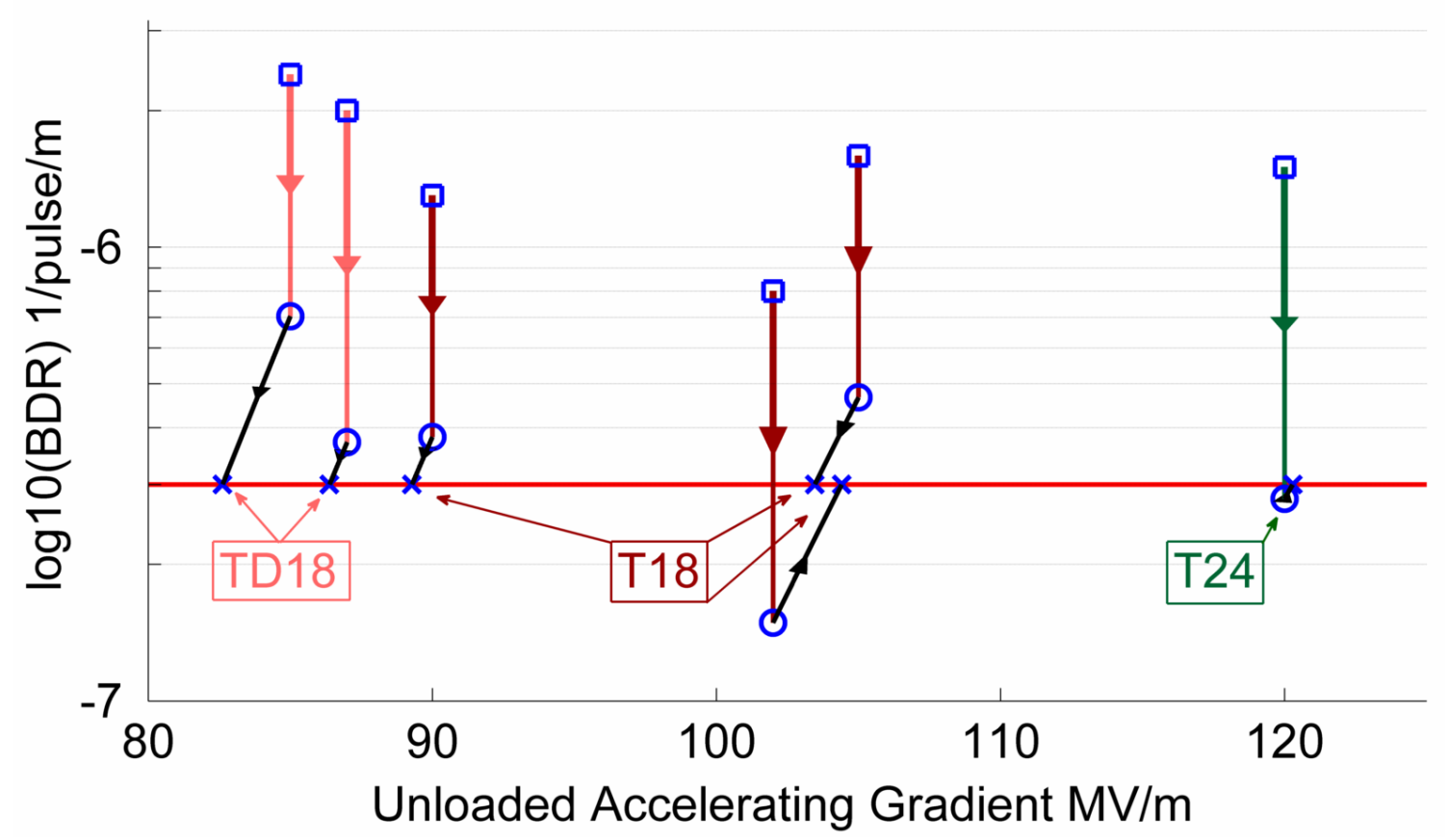

Figure 2: Gradient and breakdown rate achieved with different CLIC structures [5]. The actual measurements are marked with squares, the expected breakdown rate for the nominal pulse length with circles and the expected gradient for the nominal breakdown rate with crosses.

it is not understood why the third CERN-built structure performs less well. The two TD18 achieved an average gradient of about $87 \mathrm{MV} / \mathrm{m}$. The T24 achieved an unloaded gradient above $120 \mathrm{MV} / \mathrm{m}$, while first tests of TD24 structures are currently underway. The input power for the $120 \mathrm{MV} / \mathrm{m}$ unloaded gradient in T24 is the same as for $100 \mathrm{MV} / \mathrm{m}$ in the loaded case. A dedicated experiment in planned in CTF3 to verify the breakdown rate for the latter, which might be lower than unloaded since the power flow in the structure is reduced.

\section{Two Beam Scheme}

The RF frequency of the drive beam accelerator (DBA) is $1 \mathrm{GHz}$. The injector produces a $140 \mu$ s-long electron beam pulse; switching from filling odd to filling even buckets (and vice versa) every $240 \mathrm{~ns}$. Either a thermionic or an RF source could be used. The DBA accelerates the beam to about $2.4 \mathrm{GeV}$ with an RF to beam efficiency of $97 \%$. An $0.5 \mathrm{GHz}$ RF deflector separates the 240ns-long sub-pulses and sends every other into a delay loop, so that its bunches can be interleaved with those of the next un-delayed sub-pulse. This produces a sequence of $240 \mathrm{~ns}$-long sub-pulses spaced by 240 ns-long gaps. Three of these sub-pulses are merged in the first combiner ring and subsequently four of the new sub-pulses in the second. Thus each of the 24 final sub-pulse have 24times the initial current and only $2.5 \mathrm{~cm}$ bunch spacing. Each will feed one drive beam decelerator in the main linac. This scheme allows a total compression of the drive beam power by a factor 576 .

To demonstrate the two-beam scheme, CTF3 has been constructed and commissioned at CERN; the layout is shown in Fig. 3 and fundamental parameters in table 2. CTF3 consists of a drive beam 


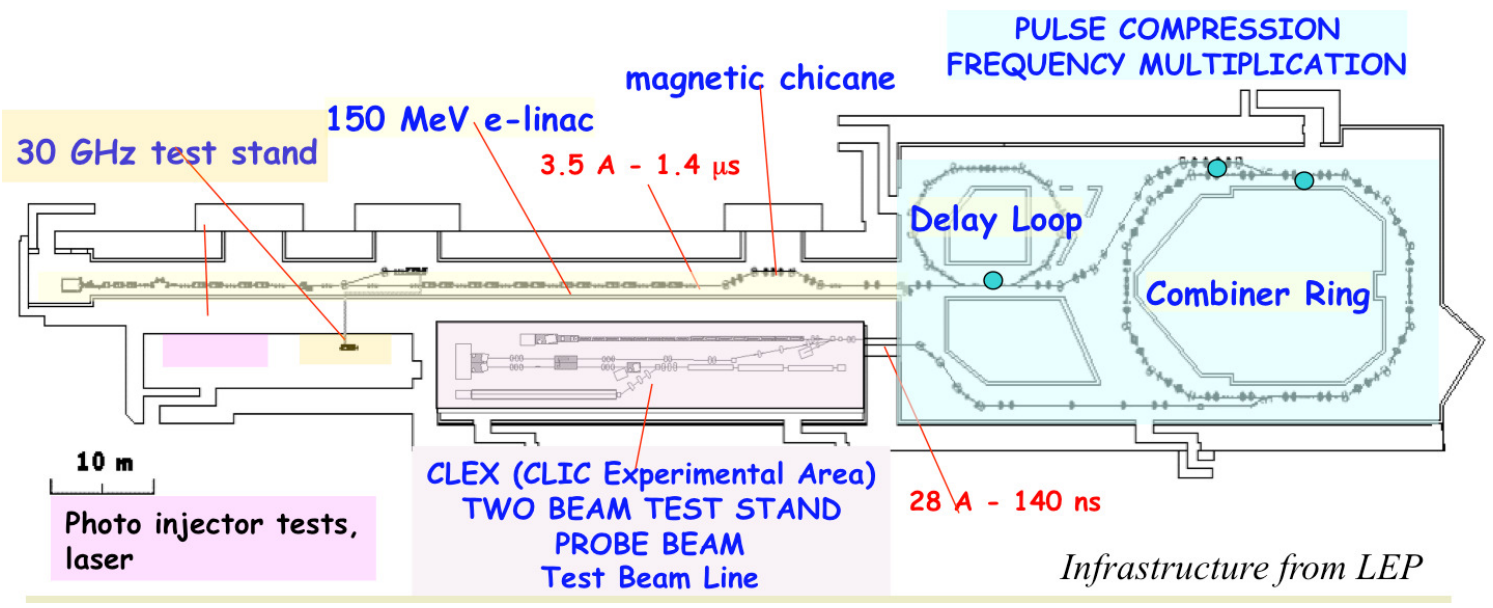

Figure 3: Layout of CTF3.

Table 2: Typical CLIC and CTF3 drive beam parameters.

\begin{tabular}{|c|c|c|c|}
\hline parameter & unit & CLIC & CTF3 \\
\hline accelerated current & $\mathrm{A}$ & 4.2 & 3.5 \\
combined current & $\mathrm{A}$ & 101 & 28 \\
final energy & $\mathrm{MeV}$ & 2400 & $\approx 120$ \\
accelerated pulse length & $\mu \mathrm{s}$ & 140 & 1.2 \\
final pulse length & $\mathrm{ns}$ & 240 & 140 \\
acceleration frequency & $\mathrm{GHz}$ & 1 & 3 \\
final bunch frequency & $\mathrm{GHz}$ & 12 & 12 \\
\hline
\end{tabular}

source, the drive beam accelerator operating at $3 \mathrm{GHz}$, the delay loop and one combiner ring. This allows to increase the initial beam current by a factor eight. The produced drive beam can be used in the two-beam test (TBTS) stand, which also includes a probe beam that simulates the CLIC main beam. Alternatively it can be sent into the test beam line (TBL), which is a small decelerator.

\subsection{Drive Beam Production}

The drive beam accelerator of CTF3 accelerates routinely a current of about $3.5 \mathrm{~A}$. It has shown full beam-loading, in which case $95 \%$ of the RF that is coupled into the accelerating structure is transmitted to the beam [9]. Using the delay loop and the combiner ring, the beam combination by a factor eight has been demonstrated, yielding a current of up to 29 A [8]. Figure 4 shows the combination for a slightly smaller final current.

In CLIC, the normalized beam emittance targets at the entrance of the decelerator are $\varepsilon_{x, y} \leq$ $150 \mu \mathrm{m}$. At the end of the CTF3 linac one routinely achieves $\varepsilon_{x, y} \approx 50 \mu \mathrm{m}$ [10]. This confirms that drive beam accelerator wakefield effects are small as predicted by simulations. After the combination system a single bunch emittance of $\varepsilon_{x, y} \approx 60 \mu \mathrm{m}$ has been observed for a single turn in the combiner ring. For more turns the beam emittance increases in the horizontal plane up to $140 \mu \mathrm{m}$ 


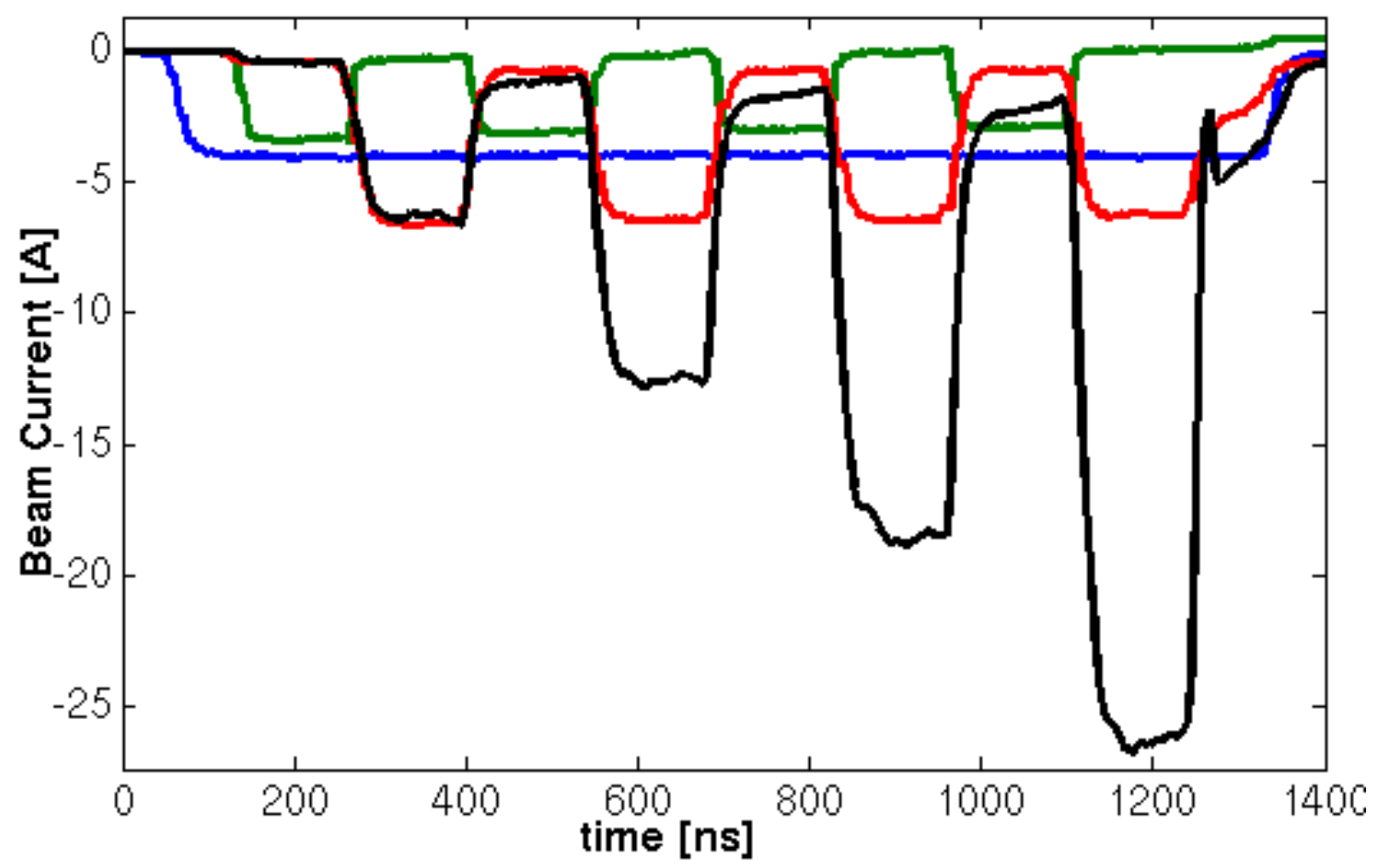

Figure 4: The drive beam combination by a factor eight in CTF3. The blue, green and red line show the current before, in and after the delay loop. The black line is the current in the combiner ring, showing the build-up turn by turn.

and the bunches that made a different number of turns in the combiner ring are not on the same orbit, which increases the emittance of the total pulse. It is planned to tune the ring to remove this problem in the coming months [10].

CLIC has very tight requirements for the phase and amplitude stability of the drive beam. In the DBA, the RF RMS phase jitter tolerance is $0.05^{\circ}$ for a constant error along the whole drive beam train [11] and $0.2 \%$ for the RF amplitude. In CTF3, an RF phase jitter of $0.035^{\circ}$ has been measured with respect to the external reference for a good klystron [11]. The power stability has been $0.21 \%$. The required CLIC beam current stability is $0.075 \%$. Measurements at the end of the CTF3 drive beam linac, showed an RMS pulse-to-pulse jitter of 1.5\% [11] and after adding a pulse-to-pulse feedback $0.054 \%$ [12]. The current stability during the pulse could not be measured, since the resolution of the instrumentation has not been good enough. An upper limit of about $0.2 \%$ charge variation for each $10 \mathrm{~ns}$ slice of the pulse has been found. The evaluation of the current jitter after the beam combination system remains to be done, once the operation of these systems has been optimized.

\subsection{PETS}

The 48 drive beam decelerators each contain about $150021.3 \mathrm{~cm}$-long PETS. They have an aperture of $23 \mathrm{~mm}$ and each produce about $130 \mathrm{MW}$ RF power, which feeds two main linac accelerating structures. Details can be found in [13]. 
Since individual accelerating structures or PETS could break down at a lower than nominal gradient or output power, it is mandatory to be able to switch off individual PETS or even better to control their output power. Otherwise the current in the concerned decelerator would have to be reduced to the level acceptable for the weakest structures thus severely compromising the overall performance. A mechanism has been developed to control the PETS power build-up [14], which allows to control the output of each individual PETS.

A PETS has also been tested at SLAC using klystrons; this prototype did include damping material but not the on-off mechanism. An input coupler for the klystron power had to be integrated in this PETS, which could compromise the RF performance. Since the pulse power varied during the tests, only those exceeding the CLIC target have been considered for the statistics. The average power and pulse length have been $7 \%$ and $10 \%$ higher than nominal, respectively. After some conditioning, the PETS ran for 80 hours with no breakdown. Based on this an expected breakdown rate of less than $2.4 \times 10^{-7} \mathrm{~m}^{-1}$ per pulse has been estimated [15], which is not far from the CLIC target of $1 \times 10^{-7} \mathrm{~m}^{-1}$. Significantly more testing time will be needed to more precisely determine the breakdown rate.

\subsection{Two-beam Acceleration}

The TBTS currently consists mainly of one PETS, one accelerating structure and the necessary instrumentation. A full two-beam module will be installed later, followed by a string of modules. The CTF3 drive beam generates power in the PETS and a test beam can be sent through the accelerating structure. Since the drive beam current is lower in CTF3 than in CLIC recirculation is used. A part of the output power of the PETS is injected at the PETS entrance, which seeds the produced $\mathrm{RF}$ and increases the output power at the cost of a reduced pulse length at full power.

Gradients up to $145 \mathrm{MV} / \mathrm{m}$ have been achieved in the TBTS [16]. The deceleration of the drive beam, the RF power measured and the probe beam acceleration are all consistent, also with the theoretical predictions.

\subsection{Drive Beam Decelerator}

The CLIC decelerator will decelerate the beam from $2.4 \mathrm{GeV}$ to $0.24 \mathrm{GeV}$. It is mandatory to achieve small losses and avoid any instability. Simulations of the decelerator have been performed to study the drive beam stability and the impact of static and dynamic imperfections. They show that the beam remains stable even if the wakefield damping is less efficient than expected and that alignment tolerance are less stringent than for the main linac [17].

In CTF3 a test beam line (TBL) is being constructed to test this deceleration. It contains 4 PETS and has space available for 16. In September it will be upgraded with an additional 4 PETS and early next year with another 4 .

The initial TBL beam energy $(120 \mathrm{MeV})$ is much smaller than even the final CLIC decelerator energy $(240 \mathrm{MeV})$. The resulting larger beam size will limit the maximum deceleration. The highest beam current in TBL sofar has been 19A, leading to the expected output power of $60 \mathrm{MW}$ per PETS. The beam deceleration of 10-11 MV is corresponds to the expectation [18]. The optics has been understood and the beam can be transported without losses, within the limitation of the current monitor accuracy. 


\section{Luminosity}

CLIC has very small target normalized transverse emittances, see table 3 . They are generated in the damping rings (DRs), which use strong wigglers. The emittance is a factor 7 in the horizontal vertical plane then that achieved in ATF (Accelerator Test Facility) at KEK and a factor 3 in the vertical [19]. Even with the ATF emittances, CLIC would already reach $40 \%$ of the nominal luminosity. Detailed simulation studies of the damping and intra-beam scattering in the CLIC DR lattice design show that the target performance can be reached with some margin [20]. Also other effects, e.g. electron cloud build-up and fast beam-ion instability, have been studied but cannot be covered here.

Emittance budgets have been defined for the RTML and the main linac for the design, the static and dynamic imperfections. In the BDS the beam develops tails, hence the performance budget has been defined in terms of the luminosity: with no imperfections in the BDS and the target emittance the luminosity would be $20 \%$ larger than nominal. For the whole beam transport lines from the damping rings to the collision point lattices exist. Simulations show, that the machine would yield $5 \times 10^{34} \mathrm{~cm}^{-2} \mathrm{~s}^{-1}$ with no imperfections [21].

\subsection{Survey and Beam-Based Alignment}

Transverse misalignments of the main linac and BDS components are the main source of static emittance dilution. The survey reference system consists of overlapping wires that run along the machine. The beam line elements are mounted on girders, with some sharing one girder. The girders measure the offset to the wires with sensors and can be moved with motors. The beam position can be measured with high resolution beam position monitors (BPMs) at each quadrupole. Also each accelerating structure contains a wakefield monitor [22].

The main linac performance target is a vertical emittance growth of less than $5 \mathrm{~nm}$ with a probability of $90 \%$. Simulations have been performed using a detailed model of the mechanical pre-alignment. Dispersion free steering (DFS), which minimises the orbit of the nominal beam and its difference to off-energy beams, is used to correct the dispersion by moving BPMs and quadrupoles. The structure supporting girders are aligned to the beam minimising the signal in the wakefield monitors. The performance target has been clearly met [23]. Tests of the DFS are planned in FACET [24].

The target for the BDS is to achieve $110 \%$ of the nominal luminosity with $90 \%$ probability, in presence of static imperfections and starting with beam emittances from the main linac correspoding to table 3 .

\subsection{Component Stabilisation}

The main beam is very sensitive to magnet motions in the main linac and BDS, due to site dependent ground motion or technical noise. We focused on the former, since the latter can also be addressed by component design, which we plan to do in the future. As a conservative benchmark, we use a ground motion model based on measurements of the CMS experimental hall floor [27], which includes some technical noise.

The main linac and BDS magnets are equipped with active stabilisation systems, which use motion sensors and piezo-electric actuators controlled by a local feedback/feed-forward system [28]. 
Table 3: Normalized main beam target emittances in CLIC.

\begin{tabular}{|c|c|c|}
\hline & $\varepsilon_{x}[\mathrm{~nm}]$ & $\varepsilon_{y}[\mathrm{~nm}]$ \\
\hline Damping ring exit & 500 & 5 \\
RTML exit & 600 & 10 \\
main linac exit & 660 & 20 \\
\hline
\end{tabular}

A prototype system has been developed and the transfer of the ground motion to the magnet has measured and compared to simulations with reasonable agreement. Based on the results of the first simulation studies, an improved system concept has also been developed, which will be constructed in the future. The final quadrupoles are mounted on a large concrete block that is supported by air-springs [29], since they are most sensitive to motion. The different transfer functions are implemented in the simulation code.

The beam-based orbit feedback uses an optimized controller and is based on the main linac and BDS correctors and BPMs [30]. A beam-beam feedback achieves sub-nanometre resolution by measuring the deflection in the collision.

The luminosity budget for dynamic imperfections is about 20\%. Simulations show that $13 \%$ of this budget is used assuming the calculated curve of the prototype stabilization and beam-based feedback. The improved stabilization system will loose only 3\%. It is explored whether ground motion sensors can be used at ATF2 to fully determine the beam orbit jitter pulse-to-pulse [31].

\section{Machine Protection and Operation}

A basic machine protection system concept has been developed [32]. The beam interlock system will switch the beam off, if the previous pulse has been bad or if an equipment failure is detected between pulses up to $2 \mathrm{~ms}$ before the next pulse. Very fast failures will need an inherently robust design.

One of the most critical failures is a large energy error of the main beam at the end of the main linac, e.g. due to failure of one drive beam sector [33]. The beam delivery system and the detector are protected against these failures by the energy collimation system, which has been designed with the intention to allow for the impact of a full beam train with no damage [34]; studies are still ongoing.

A first start-up procedure for the drive and main beam has also been defined based on CTF3 experience. The operation of CLIC at different centre-of-mass energies has also been explored concluding that one could expect the luminosity to scale roughly with $\sqrt{s}$ [35].

\section{Conclusion and Outlook}

The CDR will document the current CLIC conceptual design and feasibility. The first two volumes will be available as drafts end of this year and cover the machine, physics and detectors. The third volume will contain short summarizes, discuss implementation questions, and the plans 
for future work. For the project preparation phase, which should last until 2016, workplans and -packages have already been developed. After 2016, the project implementation can start.

In the past, the CLIC study has focused on the concept of a machine at $3 \mathrm{TeV}$ to prove the feasibility to reach such a high energy. Limited effort has been put into the concept of a $500 \mathrm{GeV}$ machine. It is therefore timely to develop a staged approach to the project that takes the physics findings, in particular of the LHC, into account.

A first stage of such a scenario could for example cover the Higgs, provided a low mass Higgs is found at the LHC, and the top, followed by two stages at higher energies to explore supersymmetry. Optimization of the machine performance, cost and schedule will be required for each stage as well as for the overall project; and a strategy will need to be devised how the physics findings can be used to define the project stages.

\section{References}

[1] H. Braun et al., CLIC-NOTE-764.

[2] D. Shulte, IPAC 2011.

[3] A. Grudiev, D. Schulte, W. Wuensch, CLIC-Note-677.

[4] A. Grudiev and W. Wuensch, Linac 2010.

[5] N. Shipman, private communication.

[6] A. Grudiev et al., PRST-AB 12,102001 (2009).

[7] T. Higo et al., IPAC 2010.

[8] P. Skowronski et al., IPAC 2011.

[9] H. Braun et al., CLIC-Note-697 (2006).

[10] R. Corsini, private communication.

[11] D. Schulte et al., LINAC 2010.

[12] G. Sterbini, private communication.

[13] I. Syratchev et al. PAC 2007.

[14] I. Syratchev, A. Cappelletti, IPAC 2010.

[15] A. Capelletti et al., submitted to NIM A XB-10 special issue, 2010.

[16] W. Farabolini, IPAC 2011.

[17] E. Adli, CERN-THESIS-2010-024.

[18] S. Doebert, private communication.

[19] Y. Honda et al, Phys. Rev. Lett. 92, 054802 (2004)

[20] Y. Papaphilippou, IPAC 2011.

[21] A. Latina, private communication.

[22] F. Pauger et al., LINAC 2010.

[23] D. Schulte, PAC 2009. 
[24] J. Amann et al., SLAC-R-930.

[25] B. Dalena et al., to be published.

[26] H. Mainaud Durand, IPAC 2011.

[27] A. Kuzmin et al., CERN EDMS 1027459 (2009).

[28] C. Collette et al., NIM A 621 (71-78), 2010.

[29] A. Gaddi et al. LCD-2010-011.

[30] J. Snuverink et al., IPAC 2011.

[31] Y. Renier et al., CLIC-Note-888 (2011).

[32] M. Joncker et al., IPAC 2010.

[33] D. Schulte and F. Zimmermann. PAC 2001.

[34] J. Resta-Lopez, PAC09

[35] D. Schulte et al., IPAC 2010. 